\title{
Photoelectric detection of polychromatic light
}

\author{
H. J. Kimble and L. Mandel* \\ Department of Physics, University of Texas at Austin, Austin, Texas 78712
}

(Received 12 April 1984)

\begin{abstract}
The problem of photoelectric detection of light is reexamined when the light is polychromatic and not strictly stationary, and when the frequency response of the detector has to be taken into account. It is shown that, whereas the one-photoelectron detection probability can always be expressed in a familiar form involving the expected light intensity, the same is not true of the twofold detection probability. In general, the photodetector does not count photons in any precise sense.
\end{abstract}

\section{INTRODUCTION}

The problem of photoelectric detection of light by a mechanism that causes an electron to make a transition from a bound state to a continuum state has already been treated several times in the past. Some of the earlier calculations were semiclassical, ${ }^{1-4}$ but several treatments in which the field is quantized have also been given, ${ }^{5-8}$ and a close correspondence between the answers obtained by the two approaches was found when the relevant dynamical variables are put into normal order. ${ }^{9-11}$

A first objective of most calculations has been an expression for the differential probability $P_{1}$ of detecting a photoelectron in some short time interval between $t$ and $t+\Delta t_{1}$ or, more generally, for the joint probability $P_{n}$ of detecting one photoelectron between $t_{1}$ and $t_{1}+\Delta t_{1}$, one photoelectron between $t_{2}$ and $t_{2}+\Delta t_{2}$, etc., and one between $t_{n}$ and $t_{n}+\Delta t_{n}$. In most calculations, but not all, it was assumed that the field is of narrow bandwidth or quasimonochromatic, and that the time intervals $\Delta t_{1}, \Delta t_{2}, \ldots, \Delta t_{n}$, although short, are very long compared with typical optical periods. The usual and widely used result, which was first obtained by Glauber, ${ }^{5}$ is that

$$
\begin{aligned}
& P_{1}=\eta\langle\hat{I}(t)\rangle \Delta t, \\
& P_{2}=\eta^{2}\left\langle: \hat{I}\left(t_{1}\right) \hat{I}\left(t_{2}\right):\right\rangle \Delta t_{1} \Delta t_{2},
\end{aligned}
$$

etc. Here $\hat{I}(t)$ is the intensity operator of the incident electromagetic field that is taken to be effectively free, ${ }^{12} \eta$ represents the detection efficiency of the detector, and : : specifies normal ordering. However, these simple expressions need to be modified when the optical field is polychromatic, with a substantial bandwidth over which the detector response varies. The required modification was already given by Glauber, ${ }^{5}$ who showed that the correlation function entering into the expression for $P_{n}$ has to be convolved with a certain response function of the detector. This problem was further discussed by Rousseau, ${ }^{8}$ who also examined the effect on the integral counting probabilities. Finite bandwidth effects were also discussed to some extent in Ref. 3.

In the following we return to this problem of the photoelectric detection of polychromatic light, and we carry the calculation of the response function further with the help of some reasonable assumptions about the nature of the detector. For simplicity, we limit our discussion to onefold and twofold probabilities $P_{1}$ and $P_{2}$, because these are the quantities which are most commonly involved in measurements, but the generalization to $P_{n}$ is readily made. We show that when the field is at least quasistationary, Eq. (1) remains valid no matter how great the bandwidth of the light may be, but the expressions for the higher-order probabilities like $P_{2}$ can no longer be put in the form of Eq. (2). We compare our results with the conclusions recently reached by Cook $^{13}$ concerning the rate of counting of a photon counter under polychromatic illumination, and we show that the detector does not count the photon flux. Finally, we examine the unusual case of a narrow-bandwidth detector with incident light of Gaussian statistics, and we demonstrate that there are no unusually large response fluctuations, as has sometimes been suggested. ${ }^{8}$

\section{THE DETECTION PROBABILITIES}

We consider an idealized detector consisting of $M$ bound electrons, that are acted on at time $t$ by a quantum field in the form of an approximately plane wave, such that each electron sees the same field. We take the initial state of a typical electron to be an eigenstate $\left|\Psi_{0}\right\rangle$ of the unperturbed electron energy $\hat{H}_{e}$,

$$
\widehat{H}_{e}\left|\Psi_{0}\right\rangle=E_{0}\left|\Psi_{0}\right\rangle, \quad E_{0}<0
$$

in which $E_{0}$ is a negative energy eigenvalue of order $1 \mathrm{eV}$. The state of the field is characterized by an arbitrary density operator $\hat{\rho}_{F}(t)$, and we take the density operator of the combined state of electron and field to be a direct product

$$
\hat{\rho}(t)=\hat{\rho}_{F}(t) \otimes\left|\Psi_{0}\right\rangle\left\langle\Psi_{0}\right| .
$$

The different electrons are assumed not to interact with each other appreciably, so that we may consider the action of the field on one electron at a time.

We shall take the interaction between the electron and the field to be given by the minimal coupling Hamiltonian, which has the form in the interaction picture, when the dipole approximation is made and the $\hat{A}^{2}$ term is discarded, 


$$
\begin{aligned}
\hat{H}_{I}\left(t^{\prime}\right)=- & \frac{e}{m} e^{i \hat{H}_{e}\left(t^{\prime}-t\right) / \hbar} \hat{\overrightarrow{\mathrm{p}}} \\
& \quad \times e^{-i \hat{H}_{e}\left(t^{\prime}-t\right) / \hbar} \cdot \hat{\overrightarrow{\mathrm{A}}}\left(\overrightarrow{\mathrm{r}}, t^{\prime}\right), t^{\prime} \geq t .
\end{aligned}
$$

$\widehat{\overrightarrow{\mathrm{p}}}$ is the electron momentum at time $t$ when the interaction is turned on, and $\vec{r}$ is the average coordinate of the bound electron, which is here taken to be a constant that may be any point in the photoelectric surface. $\widehat{\vec{A}}(\vec{r}, t)$ is the transverse vector potential of the incident electromagnetic field in the Coulomb gauge. It can be decomposed into its positive and negative frequency parts with the help of a mode expansion, and we shall write

$$
\widehat{\overrightarrow{\mathbf{A}}}(\overrightarrow{\mathbf{r}}, t)=\widehat{\overrightarrow{\mathbf{A}}}^{(+)}(\overrightarrow{\mathrm{r}}, t)+\widehat{\overrightarrow{\mathbf{A}}}^{(-)}(\overrightarrow{\mathrm{r}}, t) .
$$

We can use time-dependent perturbation theory to cal- culate the probability that the electron makes a transition at time $t+\Delta t$ from the initial state to some final freeelectron state $|\Psi\rangle$, which is an energy eigenstate in the continuum with positive eigenvalue $E$,

$$
\hat{H}_{e}|\Psi\rangle=E|\Psi\rangle, \quad E>0 \text {. }
$$

$\Delta t$ is a short time interval, which is, however, many optical periods long. Next we sum over all states of positive energy $E$, and over all other variables $\Omega$ that determine the electron state, with the help of some sensitivity function $g(E, \Omega)$ that characterizes the detector, in order to obtain the probability $P_{1}(\overrightarrow{\mathrm{r}}, t+\Delta t)$ that a photoelectron is detected and registered over the time interval from $t$ to $t+\Delta t$. This probability is increased $M$-fold when $M$ independent electrons are available for photoemission. The well-known result of such a calculation, when only energy-conserving terms are retained, is ${ }^{5,7,8}$

$$
P_{1}(\overrightarrow{\mathrm{r}}, t+\Delta t)=\int_{0}^{\Delta t} d t^{\prime} \int_{0}^{\Delta t} d t^{\prime \prime}\left\langle\hat{A}_{i}^{(-)}\left(\overrightarrow{\mathrm{r}}, t+t^{\prime}\right) \hat{A}_{j}^{(+)}\left(\overrightarrow{\mathrm{r}}, t+t^{\prime \prime}\right)\right\rangle k_{j i}\left(t^{\prime}-t^{\prime \prime}\right)
$$

for the probability of a single detection at a point $\vec{r}$ in the field, and

$$
\begin{aligned}
& P_{2}\left(\overrightarrow{\mathrm{r}}_{1}, t_{1}+\Delta t_{1} ; \overrightarrow{\mathrm{r}}_{2}, t_{2}+\Delta t_{2}\right) \\
& \quad=\int_{0}^{\Delta t_{1}} d t^{\prime} \int_{0}^{\Delta t_{2}} d t^{\prime \prime} \int_{0}^{\Delta t_{2}} d t^{\prime \prime \prime} \int_{0}^{\Delta t_{1}} d t^{\prime \prime \prime \prime}\left\langle\hat{A}_{i}^{(-)}\left(\overrightarrow{\mathrm{r}}_{1}, t_{1}+t^{\prime}\right) \hat{A}_{l}^{(-)}\left(\overrightarrow{\mathrm{r}}_{2}, t_{2}+t^{\prime \prime}\right)\right. \\
& \left.\quad \times \hat{A}_{m}^{(+)}\left(\overrightarrow{\mathrm{r}}_{2}, t_{2}+t^{\prime \prime \prime}\right) \hat{A}_{j}^{(+)}\left(\overrightarrow{\mathrm{r}}_{1}, t_{1}+t^{\prime \prime \prime \prime}\right)\right\rangle k_{j i}^{(1)}\left(t^{\prime}-t^{\prime \prime \prime \prime}\right) k_{m l}^{(2)}\left(t^{\prime \prime}-t^{\prime \prime \prime}\right)
\end{aligned}
$$

for the twofold joint probability of a detection at $\vec{r}_{1}$ over a time interval from $t_{1}$ to $t_{1}+\Delta t_{1}$, and at $\vec{r}_{2}$ over a time interval from $t_{2}$ to $t_{2}+\Delta t_{2}$ with two detectors. Both $P_{1}$ and $P_{2}$ are differential probabilities $\left(P_{1}<<1, P_{2}<<1\right)$, from which integral probabilities can be constructed. Here $k_{j i}(\tau)$ is the response function of the detector, which may be different for each detector, and is given by

$$
k_{j i}(\tau) \equiv M\left[\frac{e}{m \hbar}\right]^{2} \int_{0}^{\infty} d E \int d \Omega \sigma(E, \Omega) g(E, \Omega)\left\langle\Psi(E, \Omega)\left|\hat{p}_{j}\right| \Psi_{0}\right\rangle\left\langle\Psi_{0}\left|\hat{p}_{i}\right| \Psi(E, \Omega)\right\rangle e^{-i\left(E-E_{0}\right) \tau / \hbar}
$$

and $\sigma(E, \Omega)$ is the density of final electron states. In general, the probability $P_{n}$ involves a correlation function of the field of order $2 n$, with the operators written in normal order. As in most treatments, the field is taken to be effectively free so that time ordering of the operators appearing in Eq. (9) is not necessary. 5,6

Some simplifications are possible with the help of a few additional assumptions. Let us suppose that the final state of the electron $|\Psi(E, \Omega)\rangle$ is almost a free-particle state. Then, if we ignore internal variables like spin, the final state is characterized only by the direction of motion of the electron, besides the energy $E$. Let the incident electromagnetic field be propagating in the $z$ direction, with the vector potential $\overrightarrow{\mathrm{A}}$ in the $x y$ plane. Let $\theta$ and $\phi$ be the polar and azimuthal angles of the electron velocity, which we now identify with $\Omega$. Then the integral over $\Omega$ becomes an integral over all directions $\theta, \phi$. Let us suppose that $\sigma(E, \Omega)=\sigma(E)$, independent of direction, and that the sensitivity function $g$ has axial symmetry, so that $g(E, \Omega)=g(E, \theta)$. Then if we approximate the final state $|\Psi(E, \Omega)\rangle$ by the free-particle state with wave function

$$
\frac{1}{(2 \pi \hbar)^{3 / 2}} e^{i \overrightarrow{\mathrm{P}} \cdot \overrightarrow{\mathrm{r}} / \hbar}
$$

we obtain

$$
\begin{aligned}
\left\langle\Psi(E, \Omega)|\hat{\overrightarrow{\mathrm{p}}}| \Psi_{0}\right\rangle & \approx-\frac{\hbar}{i(2 \pi \hbar)^{3 / 2}} \int d \overrightarrow{\mathrm{r}}\left(\vec{\nabla} e^{i \overrightarrow{\mathrm{P}} \cdot \overrightarrow{\mathrm{r}} / \hbar}\right) \Psi_{0}(\overrightarrow{\mathrm{r}}) \\
& =\overrightarrow{\mathrm{P}} \widetilde{\Psi}_{0}(\overrightarrow{\mathrm{P}}), \text { with } P^{2} / 2 m=E
\end{aligned}
$$

Here $\Psi_{0}(\vec{r})$ is the Schrödinger wave function of the initial bound electron state, and $\widetilde{\Psi}_{0}(\overrightarrow{\mathbf{P}})$ is its three-dimensional Fourier transform. The momentum range of $\widetilde{\Psi}_{0}(\overrightarrow{\mathbf{P}})$ depends on the width $\delta z$ of the potential well that initially confines the electron, and, when converted to units of energy $E=P^{2} / 2 m$, is of order $\hbar^{2} /(\delta z)^{2} m$, which can be many electron volts. $\left|\widetilde{\Psi}_{0}(\overrightarrow{\mathbf{P}})\right|$ is therefore expected to be a slowly varying function of the electron energy over a range of order $1 \mathrm{eV}$. For simplicity, we shall take $\widetilde{\Psi}_{0}(\overrightarrow{\mathrm{P}})$ to be approximately axially symmetric also, so that $\widetilde{\Psi}_{0}(\overrightarrow{\mathbf{P}})=\widetilde{\Psi}_{0}(P, \theta)$.

As the electromagnetic field vector $\widehat{\overrightarrow{\mathrm{A}}}(\overrightarrow{\mathrm{r}}, t)$ was taken to lie in the $x y$ plane, we need to determine $k_{i j}(\tau)$ only for $i, j=x, y$. From Eqs. (10) and (11) we readily find 


$$
\left[\begin{array}{l}
k_{x x}(\tau) \\
k_{x y}(\tau) \\
k_{y y}(\tau)
\end{array}\right)=M\left(\frac{e}{m \hbar}\right)^{2} \int_{0}^{\infty} d E \int_{0}^{\pi} d \theta \int_{0}^{2 \pi} d \phi \sigma(E) g(E, \theta) 2 m E\left|\widetilde{\Psi}_{0}(P, \theta)\right|^{2} e^{-i\left(E-E_{0}\right) \tau / \hbar} \sin ^{3} \theta\left[\begin{array}{c}
\cos ^{2} \phi \\
\cos \phi \sin \phi \\
\sin ^{2} \phi
\end{array}\right),
$$

so that

$$
\begin{aligned}
& k_{x y}(\tau)=0, \\
& k_{x x}(\tau)=k_{y y}(\tau) \equiv k(\tau)=2 \pi \frac{M e^{2}}{m \hbar^{2}} \int_{0}^{\infty} d E \int_{0}^{\pi} d \theta E \sigma(E) g(E, \theta)\left|\widetilde{\Psi}_{0}(P, \theta)\right|^{2} \sin ^{3} \theta e^{-i\left(E-E_{0}\right) \tau / \hbar} .
\end{aligned}
$$

This may also be expressed in the form

$$
k(\tau)=\frac{1}{2 \pi} \int_{0}^{\infty} \frac{d E}{\hbar} K(E / \hbar) e^{-i\left(E-E_{0}\right) \tau / \hbar},
$$

where we have written

$$
K(E / \hbar) \equiv(2 \pi)^{2} \frac{M e^{2}}{m \hbar^{2}} \int_{0}^{\pi} d \theta E \sigma(E) g(E, \theta)\left|\widetilde{\Psi}_{0}(P, \theta)\right|^{2} \sin ^{3} \theta
$$

for the response function of the photoelectric detector to light of frequency $\omega=E / \hbar$. It is implicit in the definition that $E>0$, and that $K$ vanishes for negative $E$. We have already seen that $\left|\widetilde{\Psi}_{0}(P, \theta)\right|^{2}$ is expected to vary slowly with $E$ over the optical range. Similarly, $\sigma(E)$ and $g(E, \theta)$ are generally slowly varying functions of $E$. It follows that $K(E / \hbar)$ is expected to be a slowly varying function of frequency over the optical range, and its Fourier transform $k(\tau)$ will have a range of only a few optical periods. On making the change of variables $t^{\prime}-t^{\prime \prime}=\tau$ in Eq. (8) and $t^{\prime}-t^{\prime \prime \prime \prime}=\tau^{\prime}$ and $t^{\prime \prime}-t^{\prime \prime \prime}=\tau^{\prime \prime}$ in Eq. (9), and recognizing that $k(\tau)$ has a range that is generally much shorter than any measurable time interval $\Delta t$, we see that the limits on the $\tau, \tau^{\prime}$, and $\tau^{\prime \prime}$ integrals may be replaced by $-\infty$ to $\infty$ to a good approximation. Equations (8) and (9) then become

$$
\begin{aligned}
& P_{1}(\overrightarrow{\mathrm{r}}, t+\Delta t)=\int_{0}^{\Delta t} d t^{\prime} \int_{-\infty}^{\infty} d \tau \Gamma_{i i}^{(1,1)}\left(\overrightarrow{\mathrm{r}}, t+t^{\prime} ; \overrightarrow{\mathrm{r}}, t+t^{\prime}-\tau\right) k(\tau), \\
& P_{2}\left(\overrightarrow{\mathrm{r}}_{1}, t_{1}+\Delta t_{1} ; \overrightarrow{\mathrm{r}}_{2}, t_{2}+\Delta t_{2}\right) \\
& \quad=\int_{0}^{\Delta t_{1}} d t^{\prime} \int_{0}^{\Delta t_{2}} d t^{\prime \prime} \int_{-\infty}^{\infty} \int_{-\infty}^{\infty} d \tau^{\prime} d \tau^{\prime \prime} \Gamma_{i j j i}^{(2,2)}\left(\overrightarrow{\mathrm{r}}_{1}, t_{1}+t^{\prime} ; \overrightarrow{\mathrm{r}}_{2}, t_{2}+t^{\prime \prime} ; \overrightarrow{\mathrm{r}}_{2}, t_{2}+t^{\prime \prime}-\tau^{\prime \prime} ; \overrightarrow{\mathrm{r}}_{1}, t_{1}+t^{\prime}-\tau^{\prime}\right) k^{(1)}\left(\tau^{\prime}\right) k^{(2)}\left(\tau^{\prime \prime}\right),
\end{aligned}
$$

where we have introduced the second- and fourth-order correlation functions of the vector potential operator, ${ }^{10}$

$$
\begin{aligned}
& \Gamma_{i j}^{(1,1)}\left(\overrightarrow{\mathrm{r}}_{1}, t_{1} ; \overrightarrow{\mathrm{r}}_{2}, t_{2}\right) \equiv\left\langle\hat{A}_{i}^{(-)}\left(\overrightarrow{\mathrm{r}}_{1}, t_{1}\right) \hat{A}_{j}^{(+)}\left(\overrightarrow{\mathrm{r}}_{2}, t_{2}\right)\right\rangle, \\
& \Gamma_{i j k l}^{(2,2)}\left(\overrightarrow{\mathrm{r}}_{1}, t_{1} ; \overrightarrow{\mathrm{r}}_{2}, t_{2} ; \overrightarrow{\mathrm{r}}_{3}, t_{3} ; \overrightarrow{\mathrm{r}}_{4}, t_{4}\right) \equiv\left\langle\hat{A}_{i}^{(-)}\left(\overrightarrow{\mathrm{r}}_{1}, t_{1}\right) \hat{A}_{j}^{(-)}\left(\overrightarrow{\mathrm{r}}_{2}, t_{2}\right) \hat{A}_{k}^{(+)}\left(\overrightarrow{\mathrm{r}}_{3}, t_{3}\right) \hat{A}_{l}^{(+)}\left(\overrightarrow{\mathrm{r}}_{4}, t_{4}\right)\right\rangle .
\end{aligned}
$$

\section{QUASISTATIONARY FIELDS}

The expressions for $P_{1}$ and $P_{2}$ simplify if the field is strictly stationary. However, frequently one is interested in studying excitation and decay processes in atomic systems when there is no stationarity. Nevertheless, over the short time interval $\Delta t$ the field can usually be regarded as approximately stationary, even though the statistical properties evolve over longer times. We shall refer to such processes as quasistationary. For a quasistationary field, the correlation functions become functions of the differences between the time arguments, when these differences are very small.

For the second-order correlations, it is convenient to introduce the normalized correlation function

$$
\gamma_{i j}^{(1,1)}(t, \tau) \equiv \frac{\Gamma_{i j}^{(1,1)}(\overrightarrow{\mathrm{r}}, t ; \overrightarrow{\mathrm{r}}, t+\tau)}{\left[\left\langle\hat{I}_{i}(\overrightarrow{\mathrm{r}}, t)\right\rangle\left\langle\hat{I}_{j}(\overrightarrow{\mathrm{r}}, t+\tau)\right\rangle\right]^{1 / 2}},
$$

where $\left\langle\hat{I}_{i}(\overrightarrow{\mathrm{r}}, t)\right\rangle \equiv \Gamma_{i i}^{(1,1)}(\overrightarrow{\mathrm{r}}, t ; \overrightarrow{\mathrm{r}}, t)$ (no summation) is the expectation of the light intensity associated with the $i$ th component of the vector potential at $\overrightarrow{\mathrm{r}}$ at time $t$. We shall make the plausible assumption that $\gamma_{x x}^{(1,1)}(t, \tau)=\gamma_{y y}^{(1,1)}(t, \tau)$ $\equiv \gamma^{(1,1)}(t, \tau)$, and that $\gamma^{(1,1)}(t,-\tau)=\left[\gamma^{(1,1)}(t, \tau)\right]^{*}$ (the as- terisk denotes a complex conjugate) at least over a short range of $\tau$. But as the contributions to the integral in Eq. (15) all come from a very small range of $\tau$, we may formally identify $\gamma^{(1,1)}(t,-\tau)$ with $\left[\gamma^{(1,1)}(t, \tau)\right]^{*}$ for all $\tau$ without appreciable error. Then, since $\left\langle\hat{I}\left(\overrightarrow{\mathrm{r}}, t+t^{\prime}\right)\right\rangle$ $\approx\langle\hat{I}(\overrightarrow{\mathrm{r}}, t)\rangle \equiv \sum_{i}\left\langle\hat{I_{i}}(\overrightarrow{\mathrm{r}}, t)\right\rangle$ and $\gamma^{(1,1)}\left(t+t^{\prime}, \tau\right) \approx \gamma^{(1,1)}(t, \tau)$ for a quasistationary process, Eq. (15) becomes

$$
P_{1}(\overrightarrow{\mathrm{r}}, t+\Delta t)=\int_{0}^{\Delta t} d t^{\prime}\langle\hat{I}(\overrightarrow{\mathrm{r}}, t)\rangle \int_{-\infty}^{\infty} d \tau \gamma^{(1,1)}(t,-\tau) k(\tau) .
$$

We now introduce the normalized second-order spectral density $\phi^{(1,1)}$ of the quasistationary process by making a Fourier integral decomposition of $\gamma^{(1,1)}(t, \tau)$,

$$
\gamma^{(1,1)}(t, \tau) \equiv \frac{1}{2 \pi} \int_{0}^{\infty} \varphi^{(1,1)}(t, \omega) e^{-i \omega \tau} d \omega .
$$

Then, by substituting for $\gamma^{(1,1)}$ and $k(\tau)$ from Eqs. (21) and (13) and carrying out the $\tau$ integration, we obtain

$$
\begin{aligned}
& P_{1}(\overrightarrow{\mathrm{r}}, t+\Delta t) \\
& \quad=\Delta t\langle\hat{I}(\overrightarrow{\mathrm{r}}, t)\rangle \frac{1}{2 \pi} \int_{0}^{\infty} d \omega \varphi^{(1,1)}(t, \omega) K\left(\omega+E_{0} / \hbar\right) .
\end{aligned}
$$


The significance of $K\left(\omega+E_{0} / \hbar\right)$ becomes apparent immediately if we consider a field whose spectral density $\varphi^{(1,1)}(t, \omega)$ is extremely narrow and centered at frequency $\omega_{0}$. Then $\varphi^{(1,1)}(t, \omega)$ may be replaced by $\delta\left(\omega-\omega_{0}\right)$ and Eq. (22) becomes

$$
P_{1}(\overrightarrow{\mathrm{r}}, t+\Delta t)=\Delta t\langle\hat{I}(\overrightarrow{\mathrm{r}}, t)\rangle K\left(\omega_{0}+E_{0} / \hbar\right) .
$$

It follows that $K\left(\omega_{0}+E_{0} / \hbar\right)$ can be identified with the effective detection efficiency of the photodetector at frequency $\omega_{0}$. The negative frequency displacement $E_{0} / \hbar$ serves to remind us that the frequency $\omega_{0}$ has to exceed $\left|E_{0}\right| / \hbar$ before photodetection takes place. The integral in Eq. (22) evidently yields the detection efficiency $\bar{K}$ averaged over the spectral density $\varphi^{(1,1)}(t, \omega)$ of the light, or

$$
P_{1}(\overrightarrow{\mathrm{r}}, t+\Delta t)=\Delta t\langle\hat{I}(\overrightarrow{\mathrm{r}}, t)\rangle \bar{K}(t),
$$

no matter what the spectral width may be.

The light intensity $\langle\hat{I}(\overrightarrow{\mathrm{r}}, t)\rangle$ that appears in the equations is the intensity associated with the vector potential, because we started from a $-(e / m) \hat{\overrightarrow{\mathrm{p}}} \cdot \hat{\overrightarrow{\mathrm{A}}}$ interaction. Had we taken the interaction to be of the form $-e \hat{\overrightarrow{\mathrm{r}}} \cdot \widehat{\overrightarrow{\mathrm{E}}}$, for example, the intensity would have been that associated with the electric field, and the expression for $K\left(\omega+E_{0} / \hbar\right)$ would have been slightly different. For a quasimonochromatic field of frequency $\omega_{0}$ the connection between the two light intensities is

$$
\omega_{0}^{2}\left\langle\hat{I}_{A}(\overrightarrow{\mathrm{r}}, t)\right\rangle=\left\langle\hat{I}_{E}(\overrightarrow{\mathrm{r}}, t)\right\rangle
$$

$\left\langle\hat{I}_{E}(\overrightarrow{\mathrm{r}}, t)\right\rangle$, or the intensity associated with some other field vector, could have been used in Eq. (23), with an appropriate redefinition of $K\left(\omega_{0}+E_{0} / \hbar\right)$.

We now turn to Eq. (16) for the joint detection probability $P_{2}$. Once again it is convenient to introduce a normalized correlation function, this time of the fourth order, and with the assumption that time correlations for different Cartesian components are similar, to write

$$
\begin{aligned}
\gamma^{(2,2)}\left(\overrightarrow{\mathrm{r}}_{1}, t_{1} ; \overrightarrow{\mathrm{r}}_{2}, t_{2} ; \overrightarrow{\mathrm{r}}_{2}, t_{2}+\tau^{\prime \prime} ; \overrightarrow{\mathrm{r}}_{1}, t_{1}+\tau^{\prime}\right) \\
\equiv \frac{\Gamma_{i j j i}^{(2,2)}\left(\overrightarrow{\mathrm{r}}_{1}, t_{1} ; \overrightarrow{\mathrm{r}}_{2}, t_{2} ; \overrightarrow{\mathrm{r}}_{2}, t_{2}+\tau^{\prime \prime} ; \overrightarrow{\mathrm{r}}_{1}, t_{1}+\tau^{\prime}\right)}{\Gamma_{i j j i}^{(2,2)}\left(\overrightarrow{\mathrm{r}}_{1}, t_{1} ; \overrightarrow{\mathrm{r}}_{2}, t_{2} ; \overrightarrow{\mathrm{r}}_{2}, t_{2} ; \overrightarrow{\mathrm{r}}_{1}, t_{1}\right)} .
\end{aligned}
$$

Then $\gamma^{(2,2)}$ is normalized to unity when $\tau^{\prime}=0=\tau^{\prime \prime}$, and for a strictly stationary field it becomes a function only of $\tau^{\prime}, \tau^{\prime \prime}$, and $t_{2}-t_{1}$. The denominator on the right-hand side can be identified with the two-time intensity correlation function

$$
\Gamma_{i j j i}^{(2,2)}\left(\overrightarrow{\mathrm{r}}_{1}, t_{1} ; \overrightarrow{\mathrm{r}}_{2}, t_{2} ; \overrightarrow{\mathrm{r}}_{2}, t_{2} ; \overrightarrow{\mathrm{r}}_{1}, t_{1}\right)=\left\langle: \hat{I}\left(\overrightarrow{\mathrm{r}}_{1}, t_{1}\right) \hat{I}\left(\overrightarrow{\mathrm{r}}_{2}, t_{2}\right):\right\rangle
$$

With the assumption of quasistationarity over the time intervals $\Delta t_{1}$ and $\Delta t_{2}$, Eq. (16) can then be written

$$
\begin{aligned}
& P_{2}\left(\overrightarrow{\mathrm{r}}_{1}, t_{1}+\Delta t_{1} ; \overrightarrow{\mathrm{r}}_{2}, t_{2}+\Delta t_{2}\right) \\
& =\int_{0}^{\Delta t_{1}} d t^{\prime} \int_{0}^{\Delta t_{2}} d t^{\prime \prime} \int_{-\infty}^{\infty} \int_{-\infty}^{\infty} d \tau^{\prime} d \tau^{\prime \prime}\left\langle: \hat{I}\left(\overrightarrow{\mathrm{r}}_{1}, t_{1}\right) \hat{I}\left(\overrightarrow{\mathrm{r}}_{2}, t_{2}+t^{\prime \prime}-t^{\prime}\right):\right\rangle k^{(1)}\left(\tau^{\prime}\right) k^{(2)}\left(\tau^{\prime \prime}\right) \\
& \quad \times \gamma^{(2,2)}\left(\overrightarrow{\mathrm{r}}_{1}, t_{1} ; \overrightarrow{\mathrm{r}}_{2}, t_{2}+t^{\prime \prime}-t^{\prime} ; \overrightarrow{\mathrm{r}}_{2}, t_{2}+t^{\prime \prime}-t^{\prime}-\tau^{\prime \prime} ; \overrightarrow{\mathrm{r}}_{1}, t_{1}-\tau^{\prime}\right)
\end{aligned}
$$

We may again choose to transform this to a frequency integral, by introducing the spectral density associated with the correlations over $\tau^{\prime}$ and $\tau^{\prime \prime}$. Let

$$
\psi^{(2,2)}\left(\overrightarrow{\mathrm{r}}_{1}, t_{1} ; \overrightarrow{\mathrm{r}}_{2}, t_{2} ; \omega_{2}, \omega_{1}\right) \equiv \int_{-\infty}^{\infty} \int_{-\infty}^{\infty} \gamma^{(2,2)}\left(\overrightarrow{\mathrm{r}}_{1}, t_{1} ; \overrightarrow{\mathrm{r}}_{2}, t_{2} ; \overrightarrow{\mathrm{r}}_{2}, t_{2}+\tau^{\prime \prime} ; \overrightarrow{\mathrm{r}}_{1}, t_{1}+\tau^{\prime}\right) e^{i\left(\omega_{2} \tau^{\prime \prime}+\omega_{1} \tau^{\prime}\right)} d \tau^{\prime} d \tau^{\prime \prime}
$$

or

$$
\gamma^{(2,2)}\left(\overrightarrow{\mathrm{r}}_{1}, t_{1} ; \overrightarrow{\mathrm{r}}_{2}, t_{2} ; \overrightarrow{\mathrm{r}}_{2}, t_{2}+\tau^{\prime \prime} ; \overrightarrow{\mathrm{r}}_{1}, t_{1}+\tau^{\prime}\right)=\frac{1}{(2 \pi)^{2}} \int_{0}^{\infty} \int_{0}^{\infty} \psi^{(2,2)}\left(\overrightarrow{\mathrm{r}}_{1}, t_{1} ; \overrightarrow{\mathrm{r}}_{2}, t_{2} ; \omega_{2}, \omega_{1}\right) e^{-i\left(\omega_{2} \tau^{\prime \prime}+\omega_{1} \tau^{\prime}\right)} d \omega_{1} d \omega_{2}
$$

With the help of Eq. (13), and integration with respect to $\tau^{\prime}$ and $\tau^{\prime \prime}$, Eq. (28) then leads to

$$
\begin{array}{r}
P_{2}\left(\overrightarrow{\mathrm{r}}_{1}, t_{1}+\Delta t_{1} ; \overrightarrow{\mathrm{r}}_{2}, t_{2}+\Delta t_{2}\right)=\int_{0}^{\Delta t_{1}} d t^{\prime} \int_{0}^{\Delta t_{2}} d t^{\prime \prime}\left\langle: \hat{I}\left(\overrightarrow{\mathrm{r}}_{1}, t_{1}\right) \hat{I}\left(\overrightarrow{\mathrm{r}}_{2}, t_{2}+t^{\prime \prime}-t^{\prime}\right):\right\rangle \\
\times \frac{1}{(2 \pi)^{2}} \int_{0}^{\infty} \int_{0}^{\infty} d \omega_{1} d \omega_{2} \psi^{(2,2)}\left(\overrightarrow{\mathrm{r}}_{1}, t_{1} ; \overrightarrow{\mathrm{r}}_{2}, t_{2}+t^{\prime \prime}-t^{\prime} ; \omega_{2}, \omega_{1}\right) \\
\quad \times K_{1}\left(\omega_{1}+E_{0} / \hbar\right) K_{2}\left(\omega_{2}+E_{0} / \hbar\right)
\end{array}
$$

Once again we note the appearance of frequency-averaged detector efficiencies $K_{1}$ and $K_{2}$, but the averaging has to be performed with respect to the joint spectral density $\psi^{(2,2)}$. In general, there is no way in which the joint fourth-order spectral density can be expressed in terms of second-order spectral densities $\varphi^{(1,1)}(\omega)$.

An important simplification occurs when the field is quasimonochromatic with midfrequency $\omega_{0}$ and bandwidth $\Delta \omega \ll 1 / \Delta t$, when $\psi^{(2,2)}$ may be approximated by $\delta$ functions, and we may write

$$
\psi^{(2,2)}\left(\vec{r}_{1}, t_{1} ; \vec{r}_{2}, t_{2} ; \omega_{2}, \omega_{1}\right)=(2 \pi)^{2} \delta\left(\omega_{2}-\omega_{0}\right) \delta\left(\omega_{1}-\omega_{0}\right)
$$

Then Eq. (31) reduces to the well-known result,

$$
P_{2}\left(\vec{r}_{1}, t_{1}+\Delta t_{1} ; \vec{r}_{2}, t_{2}+\Delta t_{2}\right)=\Delta t_{1} \Delta t_{2}\left\langle: \hat{I}\left(\vec{r}_{1}, t_{1}\right) \hat{I}\left(\vec{r}_{2}, t_{2}\right):\right\rangle K_{1}\left(\omega_{0}+E_{0} / \hbar\right) K_{2}\left(\omega_{0}+E_{0} / \hbar\right)
$$


However, in general there is no simplification that is analogous to Eq. (24) for $P_{1}$.

\section{GAUSSIAN STATISTICS}

Under certain conditions, such as when the sources are in thermal equilibrium, or even when there is no equilibrium but the field results from a superposition of many independent contributions, the statistics of the field are Gaussian. It is well known that under these conditions all fourth-order correlations and spectral densities are expressible in terms of second-order ones. Let us see how this simplifies the expression for $P_{2}$ when the light is polarized.

With the help of the Gaussian moment theorem we have from Eq. (26)

$\gamma^{(2,2)}\left(\overrightarrow{\mathrm{r}}_{1}, t_{1} ; \overrightarrow{\mathrm{r}}_{2}, t_{2} ; \overrightarrow{\mathrm{r}}_{2}, t_{2}+\tau^{\prime \prime} ; \overrightarrow{\mathrm{r}}_{1}, t_{1}+\tau^{\prime}\right)$

$$
=\frac{\Gamma_{i i}^{(1,1)}\left(\overrightarrow{\mathrm{r}}_{1}, t_{1} ; \overrightarrow{\mathrm{r}}_{1}, t_{1}+\tau^{\prime}\right) \Gamma_{j j}^{(1,1)}\left(\overrightarrow{\mathrm{r}}_{2}, t_{2} ; \overrightarrow{\mathrm{r}}_{2}, t_{2}+\tau^{\prime \prime}\right)+\Gamma_{i j}^{(1,1)}\left(\overrightarrow{\mathrm{r}}_{1}, t_{1} ; \overrightarrow{\mathrm{r}}_{2}, t_{2}+\tau^{\prime \prime}\right) \Gamma_{j i}^{(1,1)}\left(\overrightarrow{\mathrm{r}}_{2}, t_{2} ; \overrightarrow{\mathrm{r}}_{1}, t_{1},+\tau^{\prime}\right)}{\Gamma_{i i}^{(1,1)}\left(\overrightarrow{\mathrm{r}}_{1}, t_{1} ; \overrightarrow{\mathrm{r}}_{1}, t_{1}\right) \Gamma_{j j}^{(1,1)}\left(\overrightarrow{\mathrm{r}}_{2}, t_{2} ; \overrightarrow{\mathrm{r}}_{2}, t_{2}\right)+\Gamma_{i j}^{(1,1)}\left(\overrightarrow{\mathrm{r}}_{1}, t_{1} ; \overrightarrow{\mathrm{r}}_{2}, t_{2}\right) \Gamma_{j i}^{(1,1)}\left(\overrightarrow{\mathrm{r}}_{2}, t_{2} ; \overrightarrow{\mathrm{r}}_{1}, t_{1}\right)} .
$$

Let us assume that the field is strictly stationary, and introduce normalized correlation functions as before [cf. Eq. (19)]. We also make the reasonable assumption that the field is cross-spectrally pure with respect to polarization. ${ }^{14}$ As the choice of directions for the $x$ and $y$ axes is arbitrary, we choose them so that $\left\langle\hat{I}_{x}(\vec{r})\right\rangle=\left\langle\hat{I}_{y}(\vec{r})\right\rangle$, in which case the normalized functions $\gamma_{i j}^{(1,1)}$ become independent of the Cartesian indices, and we have

$$
\frac{\Gamma_{i j}^{(1,1)}\left(\overrightarrow{\mathrm{r}}_{n}, t_{1} ; \overrightarrow{\mathrm{r}}_{m}, t_{2}\right)}{\left[\left\langle\hat{I}_{i}\left(\overrightarrow{\mathrm{r}}_{n}\right)\right\rangle\left\langle\hat{I}_{j}\left(\overrightarrow{\mathrm{r}}_{m}\right)\right\rangle\right]^{1 / 2}} \equiv \gamma_{i j}^{(1,1)}\left(\overrightarrow{\mathrm{r}}_{n}, t_{1} ; \overrightarrow{\mathrm{r}}_{m}, t_{2}\right)=\gamma^{(1,1)}\left(\overrightarrow{\mathrm{r}}_{n}, t_{1} ; \overrightarrow{\mathrm{r}}_{m}, t_{2}\right), \quad n, m=1,2
$$

We now introduce an abbreviated notation by writing

$$
\gamma^{(1,1)}\left(\overrightarrow{\mathrm{r}}_{n}, t_{1} ; \overrightarrow{\mathrm{r}}_{m}, t_{2}\right) \equiv \gamma_{n m}^{(1,1)}\left(t_{2}-t_{1}\right)
$$

Moreover, with the assumption of spatial cross-spectral purity ${ }^{14}$ we may set

$$
\gamma_{11}^{(1,1)}(\tau)=\gamma_{22}^{(1,1)}(\tau) \equiv \gamma^{(1,1)}(\tau), \quad \gamma_{12}^{(1,1)}(\tau)=\gamma_{12}^{(1,1)}(0) \gamma^{(1,1)}(\tau)
$$

Then Eq. (34) becomes

$\gamma^{(2,2)}\left(\overrightarrow{\mathrm{r}}_{1}, t_{1} ; \overrightarrow{\mathrm{r}}_{2}, t_{2} ; \overrightarrow{\mathrm{r}}_{2}, t_{2}+\tau^{\prime \prime} ; \overrightarrow{\mathrm{r}}_{1}, t_{1}+\tau^{\prime}\right)=\frac{\gamma^{(1,1)}\left(\tau^{\prime}\right) \gamma^{(1,1)}\left(\tau^{\prime \prime}\right)+\left|\gamma_{12}^{(1,1)}(0)\right|^{2} \gamma^{(1,1)}\left(t_{2}-t_{1}+\tau^{\prime \prime}\right) \gamma^{(1,1)}\left(t_{2}-t_{1}-\tau^{\prime}\right)^{*}}{1+\left|\gamma_{12}^{(1,1)}(0)\right|^{2}\left|\gamma^{(1,1)}\left(t_{2}-t_{1}\right)\right|^{2}}$,

and the fourth-order spectral density $\psi^{(2,2)}$ given by Eq. (29) simplifies to

$$
\psi^{(2,2)}\left(\overrightarrow{\mathrm{r}}_{1}, t_{1} ; \overrightarrow{\mathrm{r}}_{2}, t_{2} ; \omega_{2}, \omega_{1}\right)=\varphi^{(1,1)}\left(\omega_{1}\right) \varphi^{(1,1)}\left(\omega_{2}\right) \frac{1+\left|\gamma_{12}^{(1,1)}(0)\right|^{2} e^{i\left(\omega_{1}-\omega_{2}\right)\left(t_{2}-t_{1}\right)}}{1+\left|\gamma_{12}^{(1,1)}(0)\right|^{2}\left|\gamma^{(1,1)}\left(t_{2}-t_{1}\right)\right|^{2}}
$$

Hence Eq. (31) becomes

$$
\begin{aligned}
P_{2}\left(\overrightarrow{\mathrm{r}}_{1}, t_{1}+\Delta t_{1} ; \overrightarrow{\mathrm{r}}_{2}, t_{2}+\Delta t_{2}\right)=\int_{0}^{\Delta t_{1}} d t^{\prime} \int_{0}^{\Delta t_{2}} d t^{\prime \prime}\left\langle\hat{I}\left(\overrightarrow{\mathrm{r}}_{1}\right)\right\rangle\left\langle\hat{I}\left(\overrightarrow{\mathrm{r}}_{2}\right)\right\rangle & \\
& \times \frac{1}{(2 \pi)^{2}} \int_{0}^{\infty} \int_{0}^{\infty} d \omega_{1} d \omega_{2} \varphi^{(1,1)}\left(\omega_{1}\right) \varphi^{(1,1)}\left(\omega_{2}\right) K_{1}\left(\omega_{1}+E_{0} / \hbar\right) K_{2}\left(\omega_{2}+E_{0} / \hbar\right) \\
& \times\left[1+\left|\gamma_{12}^{(1,1)}(0)\right|^{2} e^{i\left(\omega_{1}-\omega_{2}\right)\left(t_{2}-t_{1}+t^{\prime \prime}-t^{\prime}\right)}\right]
\end{aligned}
$$

This can be reexpressed in a more transparent way. Let us consider the case of identical detectors, $K_{1}=K_{2}$, and let us denote by $\varphi_{M}^{(1,1)}(\omega)$ the normalized spectral density of second order modified by the spectral response of the detector,

$$
\varphi_{M}^{(1,1)}(\omega) \equiv \varphi^{(1,1)}(\omega) K\left(\omega+E_{0} / \hbar\right) / \bar{K},
$$

where $\bar{K}$ is defined as in Eq. (24). Similarly, let us denote by $\gamma_{M}^{(1,1)}(\tau)$ the modified normalized autocorrelation function of second order, which is the Fourier transform of $\varphi_{M}^{(1,1)}(\omega)$,

$$
\gamma_{M}^{(1,1)}(\tau) \equiv \frac{1}{2 \pi} \int_{0}^{\infty} \varphi_{M}^{(1,1)}(\omega) e^{-i \omega \tau} d \omega
$$

Then Eq. (39) can be written

$$
P_{2}\left(\overrightarrow{\mathrm{r}}_{1}, t_{1}+\Delta t_{1} ; \overrightarrow{\mathrm{r}}_{2}, t_{2}+\Delta t_{2}\right)=\left\langle\hat{I}\left(\overrightarrow{\mathrm{r}}_{1}\right)\right\rangle\left\langle\hat{I}\left(\overrightarrow{\mathrm{r}}_{2}\right)\right\rangle \bar{K}^{2} \int_{0}^{\Delta t_{1}} d t^{\prime} \int_{0}^{\Delta t_{2}} d t^{\prime \prime}\left[1+\left|\gamma_{12}^{(1,1)}(0)\right|^{2}\left|\gamma_{M}^{(1,1)}\left(t_{2}-t_{1}+t^{\prime \prime}-t^{\prime}\right)\right|^{2}\right],
$$


which illustrates rather clearly how the correlation properties of the field have been modified by the spectral response of the detector. The more nearly the frequency response $K\left(\omega+E_{0} / \hbar\right)$ of the detector is constant with frequency, the more nearly does the modified correlation function $\gamma_{M}^{(1,1)}(\tau)$ approach the original function $\gamma^{(1,1)}(\tau)$.

\section{v. PHOTON COUNTING}

From time to time the question is raised whether the photoelectric detector counts photons, i.e., whether it responds to the flux of incident photons, or to some other quantity that is not directly related to the number of photons. This question recently surfaced again in connection with an expression that was constructed by Cook $^{13}$ for the photon flux. From the foregoing it should be clear that a detector whose operation is based on the nonresonant interaction between a bound electron and the field does not count photons in any precise sense.

It is of course possible to express the differential counting probability given by Eq. (22) in a form that directly contains the spectral density of the photon flux $\Phi_{\mathrm{ph}}^{(1,1)}(\omega)$. Let us rewrite Eq. (22) when the field is stationary as

$P_{1}(\overrightarrow{\mathrm{r}}, t+\Delta t)=\Delta t \frac{1}{2 \pi} \int_{0}^{\infty} d \omega \Phi_{A}^{(1,1)}(\omega) K\left(\omega+E_{0} / \hbar\right)$,

where $\Phi_{A}^{(1,1)}(\omega)$ is the second-order (unnormalized) spectral density of the vector potential $A$. Then ${ }^{13,15}$

$$
\Phi_{A}^{(1,1)}(\omega)=\left(\frac{\hbar}{2 \omega \epsilon_{0}}\right) \Phi_{\mathrm{ph}}^{(1,1)}(\omega)
$$

and Eq. (43) becomes

$$
\begin{aligned}
& P_{1}(\overrightarrow{\mathrm{r}}, t+\Delta t) \\
& \quad=\Delta t \frac{1}{2 \pi} \int_{0}^{\infty} d \omega\left[\frac{\hbar}{2 \omega \epsilon_{0}}\right] \Phi_{\mathrm{ph}}^{(1,1)}(\omega) K\left(\omega+E_{0} / \hbar\right) .
\end{aligned}
$$

By introducing the operator

$$
\begin{array}{r}
\hat{R} \equiv c \frac{1}{L^{3}} \sum_{\overrightarrow{\mathrm{k}}, s} \sum_{\overrightarrow{\mathrm{k}}{ }^{\prime}, s^{\prime}} \hat{a}_{\overrightarrow{\mathrm{k}} s}^{\dagger} \hat{a}_{\overrightarrow{\mathrm{k}}}{ }^{\prime} \vec{\epsilon}^{\prime} \vec{\epsilon}_{\overrightarrow{\mathrm{k}} s}^{*} \cdot \epsilon_{\overrightarrow{\mathrm{k}} s^{\prime}} \\
\times e^{i\left[\left(\overrightarrow{\mathrm{k}}{ }^{\prime}-\overrightarrow{\mathrm{k}}\right) \cdot \overrightarrow{\mathrm{r}}-\left(\omega^{\prime}-\omega\right) t\right]},
\end{array}
$$

which has sometimes been taken to represent the flux density of photons, ${ }^{13,15}$ and by introducing the normalized spectral density of the photon flux density

$$
\varphi_{\mathrm{ph}}^{(1,1)}(\omega) \equiv c \Phi_{\mathrm{ph}}^{(1,1)}(\omega) /\langle\widehat{R}\rangle,
$$

we can rewrite Eq. (45)

$$
\begin{aligned}
P_{1}(\overrightarrow{\mathrm{r}}, t+\Delta t)= & \langle\hat{R}\rangle \Delta t\left(\frac{\hbar}{2 \epsilon_{0} c}\right) \frac{1}{2 \pi} \\
& \times \int_{0}^{\infty} d \omega \frac{1}{\omega} \varphi_{\mathrm{ph}}^{(1,1)}(\omega) K\left(\omega+E_{0} / \hbar\right) .
\end{aligned}
$$

Hence $P_{1}$ is proportional to $\langle\hat{R}\rangle$. However, the same average photon flux, distributed differently over the frequencies, would evidently elicit a different response from the detector, because of the change of $\varphi_{\mathrm{ph}}^{(1,1)}(\omega)$. Hence $P_{1}$ is not a direct measure of the photon flux.

On the other hand, Eq. (48) suggests that we may construct a field vector $\widehat{\overrightarrow{\mathrm{F}}}(\overrightarrow{\mathrm{r}}, t)$ such that the counting rate of the detector is directly given by the integrated spectral density of $\hat{\overrightarrow{\mathrm{F}}}(\overrightarrow{\mathrm{r}}, t)$. For this purpose we choose the positive frequency part $\widehat{\vec{F}}^{(+)}(\vec{r}, t)$ to have the plane-wave expansion

$$
\begin{gathered}
\widehat{\overrightarrow{\mathrm{F}}}^{(+)}(\overrightarrow{\mathrm{r}}, t)=\frac{1}{L^{3 / 2}} \sum_{\overrightarrow{\mathrm{k}}, s}\left[\frac{\hbar}{2 \omega \epsilon_{0}}\right]^{1 / 2} K^{1 / 2}\left(\omega+E_{0} / \hbar\right) \\
\times \hat{a}_{\overrightarrow{\mathrm{k}} s} \overrightarrow{\epsilon_{\overrightarrow{\mathrm{k}} s}} e^{i(\overrightarrow{\mathrm{k}} \cdot \overrightarrow{\mathrm{r}}-\omega t)},
\end{gathered}
$$

from which it follows that ${ }^{13,15}$

$$
\Phi_{F}^{(1,1)}(\omega)=\left(\frac{\hbar}{2 \omega \epsilon_{0}}\right) K\left(\omega+E_{0} / \hbar\right) \Phi_{\mathrm{ph}}^{(1,1)}(\omega),
$$

and therefore

$$
P_{1}(\overrightarrow{\mathrm{r}}, t+\Delta t)=\Delta t \frac{1}{2 \pi} \int_{0}^{\infty} d \omega \Phi_{F}^{(1,1)}(\omega) .
$$

However, no comparably simple expression can be written for the joint detection probability $P_{2}$ because the fourthorder spectral density, even of a strictly stationary field, depends on three frequencies.

\section{THE CASE OF A NARROW-BANDWIDTH DETECTOR}

In the foregoing treatment of the photodetector it was taken for granted that the response bandwidth of the detector is very large, or that its response time is very short compared with the measurement interval $\Delta t$. This assumption, which is reasonable in practice for almost all photodetectors, allowed us to replace a finite integral in Eq. (8) by an infinite integral in Eq. (15), and two finite integrals in Eq. (9) by two infinite integrals in Eq. (16), and it led to considerable simplification.

However, in a paper dealing with some questions relating to the response of a photodetector, ${ }^{8}$ the claim was made that when the detector response bandwidth is narrow compared with the bandwidth of the light, and when the light obeys Gaussian statistics, the detector response can exhibit very large fluctuations. In particular, the ratio $P_{2}(\overrightarrow{\mathrm{r}}, t+\Delta t ; \overrightarrow{\mathrm{r}}, t+\Delta t) / P_{1}^{2}(\overrightarrow{\mathrm{r}}, t+\Delta t)$ can become much larger than unity. This conclusion was based on somewhat different initial expressions for $P_{1}$ ard $P_{2}$, which disagree with Eqs. (8) and (9). However, because of the unusual conclusion, it seemed worthwhile to reexamine the implications of Eqs. (8) and (9) for $P_{1}$ and $P_{2}$ under these conditions also.

We assume that the field is stationary and obeys Gaussian statistics with cross-spectral purity, as before, so that all fourth-order correlations are reducible to second-order correlations $\gamma^{(1,1)}(\tau)$ with the help of the Gaussian moment theorem. We assume that we are dealing with a single detector, so that space variables can be discarded, and 
we take the spectral density to be Lorentzian, with

$$
\gamma^{(1,1)}(\tau)=e^{-\lambda|\tau|} e^{-i \omega_{0} \tau}
$$

$\lambda$ is the effective bandwidth of the light and $\omega_{0}$ is its midfrequency. In order to treat the case in which the detector has a narrow frequency response, we shall take the response function $k(\tau)$ to have a somewhat similar struc- ture, with

$$
k(\tau)=k_{0} e^{-\beta|\tau|} e^{-i \omega_{0} \tau} .
$$

The relation between $\lambda$ and $\beta$ is arbitrary.

The integrations in Eqs. (8) and (9) can then be carried out explicitly, without further approximation, and we find after some straightforward but rather lengthy manipulations,

$$
\begin{aligned}
P_{1}(t+\Delta t)=\frac{2 k_{0}\langle\hat{I}\rangle \Delta t}{\lambda+\beta}\left[1-\frac{1}{(\lambda+\beta) \Delta t}(\right. & \left.\left.1-e^{-(\lambda+\beta) \Delta t}\right)\right] \\
P_{2}(t+\Delta t ; t+\Delta t)=P_{1}^{2}(t+\Delta t)+k_{0}^{2}\langle\hat{I}\rangle^{2}[ & \frac{4 \Delta t}{\lambda \beta(\lambda+\beta)}+\frac{4 \Delta t}{(\lambda+\beta)^{3}}-\frac{2}{(\lambda \beta)^{2}}-\frac{4}{\lambda \beta(\lambda+\beta)^{2}}-\frac{10}{(\lambda+\beta)^{4}} \\
& +\frac{1}{(\lambda-\beta)^{2}}\left[\frac{2}{\lambda^{2}} e^{-2 \lambda \Delta t}+\frac{2}{\beta^{2}} e^{-2 \beta \Delta t}\right]+\frac{2}{(\lambda+\beta)^{4}} e^{-2(\lambda+\beta) \Delta t} \\
& \left.+\left[\frac{8 \Delta t}{(\lambda+\beta)^{3}}-\frac{2}{(\lambda+\beta)^{4}}-\frac{6 \lambda^{2}+6 \beta^{2}+52 \lambda \beta}{(\lambda+\beta)^{4}(\lambda-\beta)^{2}}\right] e^{-(\lambda+\beta) \Delta t}\right] .
\end{aligned}
$$

There is no singularity when $\lambda=\beta$.

It is now straightforward to calculate the ratio $P_{2} / P_{1}^{2}$ for certain combinations of $\lambda$ and $\beta$. If $\Delta t$ is sufficiently short that $\lambda \Delta t<<1$ and $\beta \Delta t<<1$ (but $\Delta t$ is still many optical periods long), we find from Eqs. (54) and (55)

$$
P_{2}(t+\Delta t ; t+\Delta t) / P_{1}^{2}(t+\Delta t)=2+O((\lambda+\beta) \Delta t)
$$

with $P_{1}$ proportional to $(\Delta t)^{2}$. On the other hand, if the detector bandwidth is much narrower than that of the light so that $\lambda \gg \beta$, and $\Delta t$ is chosen that $\lambda \Delta t \gg 1$ and $\beta \Delta t<1$, then the equations yield

$$
P_{2}(t+\Delta t ; t+\Delta t) / P_{1}^{2}(t+\Delta t)=2+O(\beta / \lambda) .
$$

with $P_{1}$ proportional to $(\Delta t)$. Both these conclusions are consistent with what one would expect from Gaussian statistics, and they show no sign of the large detector fluctuations that were thought to exist when the detector has a narrow-bandwidth response. Note also that since Eqs. (54) and (55) are invariant with respect to the interchange of $\lambda$ and $\beta$, there is a sense in which the spectral distribution of the incident light and the detector response function play equivalent roles in the detection process, at least for the special case of Gaussian light.

\section{ACKNOWLEDGMENT}

This work was supported by the National Science Foundation.
*Permanent address: Department of Physics and Astronomy, University of Rochester, Rochester, New York 14627.

${ }^{1}$ G. Wentzel, Z. Phys. 40, 574 (1926).

${ }^{2}$ L. Mandel, in Progress in Optics, edited by E. Wolf (NorthHolland, Amsterdam, 1963), Vol. II, p. 181.

${ }^{3}$ L. Mandel, E. C. G. Sudarshan, and E. Wolf, Proc. Phys. Soc. London 84, 435 (1964).

${ }^{4}$ W. E. Lamb, Jr. and M. O. Scully, in Polarisation, Matière et Rayonnement (Presses Universitaires de France, Paris, 1969), p. 363.

${ }^{5}$ R. J. Glauber, Phys. Rev. 130, 2529 (1963); and in Quantum Optics and Electronics, edited by C. deWitt, A. Blandin, and C. Cohen-Tannoudji (Gordon and Breach, New York, 1965), p. 63.

6P. C. Kelley and W. H. Kleiner, Phys. Rev. 136, A316 (1964).

${ }^{7}$ D. Meltzer and L. Mandel, Phys. Rev. 188, 198 (1969).

${ }^{8}$ M. Rousseau, J. Phys. A 8, 1265 (1975); 10, 1043 (1977).

${ }^{9}$ E. C. G. Sudarshan, Phys. Rev. Lett. 10, 277 (1963).

${ }^{10}$ R. J. Glauber, Phys. Rev. 131, 2766 (1963).

${ }^{11}$ J. R. Klauder, Phys. Rev. Lett. 16, 534 (1966); see also J. R. Klauder and E. C. G. Sudarshan, Fundamentals of Quantum Optics (Benjamin, New York, 1968).

${ }^{12} \mathrm{We}$ distinguish all Hilbert space operators by a caret.

${ }^{13}$ R. J. Cook, Phys. Rev. A 25, 2164 (1982); 26, 2754 (1982).

${ }^{14}$ L. Mandel, J. Opt. Soc. Am. 51, 1342 (1961).

${ }^{15}$ L. Mandel, Phys. Rev. 144, 1071 (1966). 